\title{
Ulfried Geuter: Praxis Körperpsychotherapie. 10 Prinzipien der Arbeit im therapeutischen Prozess
}

\author{
Springer Verlag, 2019, Berlin, 508 Seiten, 44,99€(D)
}

$\mathrm{N}$ achdem Ulfried Geuter 2015 sein erstes umfassendes theoretisches Werk zur Körperpsychotherapie im deutschsprachigen Raum vorgelegt hat, folgte nun 2019 der zweite Band als Handwerk zur körperpsychotherapeutischen Praxis. Sein Ansinnen, die vielfältigen und individuellen körperpsychotherapeutischen Landschaften mit vielen Verfahren, Methoden und Techniken in Form einer gemeinsamen Systematik zu beschreiben, ist möglicherweise von vorneherein ein schwieriges Unterfangen und könnte auch in einer mission impossible enden. Doch schon im ersten Buch scheint Geuter dies zu wissen, und er antwortet darauf mit dem Argument, dass den körperpsychotherapeutischen Schulen aufgrund der Vielfalt in ihren Interventionen durchaus eine gewisse übergreifende Systematik in der Praxis fehle. Dies ist ein gutes Argument, gerade in Zeiten, wo sich die psychotherapeutischen Schulen mehr um Gemeinsamkeiten, Integration und eine Psychotherapie bemühen und wo es schließlich $a b$ Herbst 2020 auch ein Psychotherapiestudium gibt. Gelingt es Geuter mit diesem Buch, eine gemeinsame Sprache der Körperpsychotherapie zu finden?

Als Forscher, Autor und Mensch hat er die Vielfalt und Unterschiede der verschiedensten körper(psycho)therapeutischen Methoden über Jahre hinweg theoretisch und praktisch kennengelernt und vielfach darüber publiziert. Sein Fokus hier ist nun der Versuch, die übergreifenden Gemeinsamkeiten der therapeutischen Praxis herauszuarbeiten. Er nennt diese die zehn körperpsychotherapeutischen Prinzipien. Und er führt den / die kritische Leserln auf diesem Weg in wohlbekanntes Terrain. Prüfen Sie selbst, ob Sie sich mit Ihrer körperpsychotherapeutischen Richtung darin wiederfinden.
Ich wage zu behaupten, dass wohl jede / $r$ Körperpsychotherapeutln es erwartet hätte, dass Wahr-

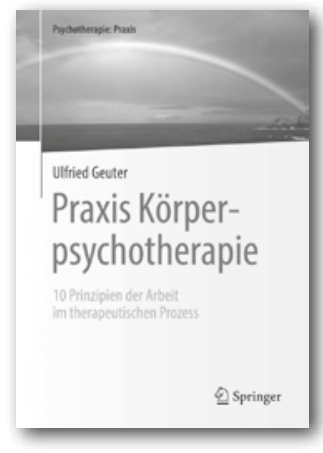
nehmen und Spüren immer am Anfang des Weges in der Therapie stehen und den Grundstock sowohl für das Erleben der eigenen Leiblichkeit als auch der Identität des Patienten darstellen. Folgerichtig ist es auch das erste Prinzip. Es ist der Zugang zum Menschen oder, wie Geuter es formuliert: „Wahrnehmen und Spüren heißt, Kontakt zu sich selbst und zur Welt aufzunehmen.“ (S. 85) In diesem ersten Prinzip wird die Struktur auch der nachfolgenden Kapitel sehr deutlich: Geuter nutzt einen beschreibenden, z.T. additiven Stil und benennt unterschiedliche Positionen von maßgeblichen Autorlnnen aus der körperpsychotherapeutischen Landschaft, schärft die unterschiedlichen Begrifflichkeiten wie z. B. Spüren und Fühlen, erläutert therapeutische Vorgehensweisen und klinische Anwendungen und gibt zahlreiche Therapiebeispiele. Für den / die Leserln wird somit körperpsychotherapeutische Arbeit sehr nachvollziehbar, begreifbar und verständlich dargestellt.

Das zweite Prinzip auf diesem Weg heißt Gewahrsein und Gegenwart. „Gewahrsein wird als der geistige Zustand vorgestellt, in dem die Aufmerksamkeit auf die Wahrnehmung gerichtet wird, um sich das zu vergegenwärtigen, was im Moment ist.“ (S.110) Noch vor einigen Jahren hätten uns die TeamkollegInnen stirnrunzelnd gefragt, was denn damit gemeint sei. Aber nachdem ja bekanntlich in den letzten Jahren die „Achtsamkeitswelle“ über die gesamte „Psychoszene“ geschwappt ist, ist es kaum mehr notwendig, solche Begriffe zu defi- 
nieren. Geuter diskutiert dieses Prinzip denn auch im letzten Abschnitt im Kontext der achtsamkeitsbasierten Psychotherapie und betont vor allem achtsames Gewahrsein.

Erkunden und Entdecken stehen für das dritte Prinzip. Hierbei werden die PatientInnen eingeladen, eine Entdeckungsreise zu sich selbst zu unternehmen, um eigener Empfindungen, Handlungen, Gedanken usw. besser gewahr zu werden und damit Möglichkeiten zu ihrer Veränderung vorzunehmen. Es geht hierbei nicht um Deutung oder Aufdeckung von Verhaltensweisen des Patienten vonseiten des Therapeuten, vielmehr nimmt dieser eine wohlwollende dialogische Haltung ein, um den Patienten zur Selbsterkundung zu bewegen. Der Hinweis, dass die aufdeckende Vorgehensweise gerade bei somatoformen Störungen, aber auch bei vielen psychosomatischen Krankheitsbildern schädlich ist, ist wichtig und wird heute nicht nur von humanistisch orientierten KollegInnen praktiziert, sondern findet zunehmend auch bei psychoanalytischen KollegInnen immer mehr Akzeptanz.

Aktivieren und Ausdrücken als das vierte Prinzip versinnbildlichen wie kaum ein anderes Prinzip sowohl die Ablehnung als auch Begeisterung für körperpsychotherapeutisches Arbeiten. Wer kennt nicht den Vorwurf der KollegInnen, dass die Patientlnnen sich in der Körpertherapie nur ausagieren. Und wer kennt nicht die tiefe Begeisterung und Bewegtheit, wenn eine Patientin berichtet, dass es ihr nach Jahren reiner Gesprächstherapie endlich gelungen sei, ihre Gefühle von Ärger, Hass und Verachtung in der Körpertherapie auszudrücken. Für mich zählen die Erläuterungen in diesem Teil des Buches zu den wertvollsten, weil Geuter mit einer prätentiösen Sicherheit theoriegeleitet das Aktivieren und Ausdrücken unterschiedlichster Gefühle an zahlreichen Beispielen vorstellt.

Im fünften Prinzip geht es um das Regulieren und Modulieren. Damit ist zum einen die Kontrolle von überschießenden emotionalen
Reaktionen gemeint, zum anderen, wie übermäßige Kontrolle und Erstarrung aufgelöst werden kann. Thematisch geht es hierbei sehr viel um Stressregulation bzw. Selbstregulation. Dieses Kapitel finde ich gerade für die klinisch arbeitenden KollegInnen sehr wichtig, weil neben der Erarbeitung einer stabilen therapeutischen Beziehung auch Tools für die PatientInnen aufgezeigt werden, wie sie ihre Emotionen lenken, dämpfen oder zurückgewinnen können.

Zentrieren und Erden sind die mit bekanntesten Techniken der Körpertherapie, die im sechsten Prinzip vorgestellt werden. Zentrieren bedeutet, den eigenen Schwerpunkt und die eigene Mitte zu finden. Erden bedeutet, den sicheren Stand zu finden, und wird in der körperpsychotherapeutischen, aber auch bewegungs- und tanztherapeutischen Arbeit häufig mit der Aufrichtung in vielen Modifikationen verbunden.

Berühren und Halten in der Therapie gehört zu den großen Vorbehalten in der Psychotherapie seit Freuds' Abstinenzgebot vor über hundert Jahren. Dessen Nutzen und Schaden wird heutzutage immer noch von Gegnern und Befürwortern intensiv diskutiert. Für mich ist dieses siebte Prinzip eines der herausragendsten, weil es Geuter gelingt, zum einen zur Berührung eine grundsätzliche Bedeutung für die menschliche Existenz aus wissenschaftlicher Sicht herauszuarbeiten, zum anderen aber auch auf die Widersprüchlichkeiten der Berührungsgegner verweist. Letztendlich löst er deren Ablehnung auf, indem er betont: „Berührung ist an sich weder hilfreich noch gefährlich. Sie kann passend oder unpassend, heilsam oder unheilsam sein, genauso wie Worte, abhängig von ihrer Intention und ihrem Kontext.“ (S. 253) Berührung wird damit zu einer wichtigen Form im psychotherapeutischen Dialog.

Dennoch will ich ein wenig Kritik üben: In der Fülle des sehr wertvoll dargestellten Wissens auch hier wieder mehr additiv - vermisse ich eine gewisse Struktur und Stringenz. Und ich 
vermisse hier maßgebliche Leibtherapeuten wie Petzold (Integrative Leibtherapie) und Glaser (Eutonie) mit ihren fundierten Ausarbeitungen zur leibbezogenen Therapie. Andererseits haben mich die sehr offenen und sorgsam dargestellten Therapiebeispiele über ausgelöste Veränderungsprozesse bei PatientInnen, aber auch der Hinweis auf persönliche Fehler (S. 278) sehr beeindruckt. Ich habe selten therapeutische Bücher mit einer vergleichbaren Nähe, Offenheit und Ehrlichkeit gelesen.

Das achte und neunte Prinzip erinnern mich sehr an die bewegungs- und tanztherapeutische Arbeit in der Körperpsychotherapie. Inszenieren und Interagieren greift Elemente aus der Gestalttherapie und des Psychodramas auf. Ausgehend vom psychodynamischen Konzept des Enactments bis hin zum Rollenspiel beschreibt Geuter die verschiedenen Formen des Dialogs, die sich im szenischen Handeln entwickeln können.

Das verkörperte Erleben ist für die therapeutische Transformation genauso wichtig wie das verbalisierte Verstehen. Das betont Geuter im neunten Prinzip und argumentiert mit wissenschaftlicher Prägnanz letztendlich dafür, dass Körperpsychotherapie neben der verbalen Therapie ein zentrales Verfahren ist. Für Geuter sind Verkörpern und Handeln wichtige Ziele in der Therapie, gerade bei Menschen, die sich von ihrem Körper entfremdet erleben, ein gestörtes Körperbild haben oder zu ihrem eigenen Körper in einer objektiven, nicht zu überwindenden Distanz stehen.

Schließlich das zehnte Prinzip: Reorganisieren bedeutet, dass ein Mensch seine Erfahrungen neu organisiert, vor allem, indem er bedeutungsvolle neue und verkörperte Erfahrungen macht. Transformieren meint hingegen, die affektmotorischen Schemata in einer Weise zu verändern, dass ein Mensch Möglichkeiten findet, ein symptomfreies und zufriedenstellenderes Leben zu führen. Das steht natürlich am Ende einer Therapie und ist letztendlich Ziel jeder Körperpsychotherapie und Psychotherapie.
Damit endet aber noch nicht das Buch, sondern Geuter benennt in weiteren Kapiteln die Indikationen und Kontraindikationen für Körperpsychotherapie, geht auf das wichtige Thema des Sprechens in der Körperpsychotherapie ein, die von vielen Kolleginnen leider immer noch als nonverbale Therapie bezeichnet wird. Schließlich führt er auf weiteren 25 Seiten die hervorragende Bedeutung der therapeutischen Beziehung auf, geht auf die Wirkfaktoren in der Psychotherapie ein, zeigt Forschungsergebnisse auf und betont, wie wichtig gerade für KörperpsychotherapeutInnen Kreativität und Lebendigkeit ist.

Warum ist dieses Buch so wertvoll? Es begleitet die Körperpsychotherapie im deutschsprachigen Raum von einer verwirrenden Vielfalt an Methoden hinaus auf den Platz eines übersichtlichen und maßgeblichen Verfahrens mit klaren Prinzipien und deren unbedingten Notwendigkeit im Rahmen einer modernen bio-psycho-sozialen Therapie. Das bedeutet für psychiatrische, psychosomatische, psychotherapeutische und Suchtkliniken ohne Körperpsychotherapie: Es herrscht ein Mangel in der Institution!

Das Buch ist so wertvoll, weil der Körper in den etablierten Psychotherapieverfahren immer noch stiefmütterlich behandelt wird. Und es ist wertvoll für viele unserer KollegInnen, um sich ein Bild über unsere Arbeit zu machen. Ulfried Geuter hat mit diesem Buch nicht nur einen unersetzlichen Beitrag für viele unserer PatientInnen und Klientlnnen geleistet, sondern ebenso einen wichtigen Beitrag für ÄrztInnen, PsychologInnen, Krankenschwestern / -pfleger, Krankenkassenträger und viele andere Berufsgruppen, die unsere Arbeit manchmal nur schwer verstehen können.

Für professionelle KörperpsychotherapeutInnen wird das Buch in den einzelnen Kapiteln sowohl Zustimmung als auch kritische Gegenrede auslösen. Vor allem aber gibt es wertvolle und beachtenswerte Denkanstöße, Anregungen und Ergänzungen zur eigenen Praxis. Für 
Auszubildende in der Körperpsychotherapie und Menschen, die das Verfahren nicht unmittelbar kennen, vermittelt das Buch einen Einblick in den Reichtum und die Vielfalt der Körperpsychotherapie. Ich denke, dass dieses Buch zukünftig in vielen Bücherregalen unterschiedlicher Berufsprofessionen stehen wird.

Für eine moderne Körperpsychotherapie und insbesondere für eine zukünftige bio-psychosoziale Therapie, die sich im zweiten Jahrzehnt des 21. Jahrhunderts besonders mit vielfach beschädigter Leiblichkeit auseinandersetzen muss, hat Geuter ein sehr wertvolles Werk geschrieben. Es wird Menschen helfen, ihren Körper wieder als einen sicheren Ort und Freund erleben zu dürfen. Es wird helfen, dass mehr Menschen in den Genuss einer körperpsychotherapeutischen Behandlung kommen und damit heilsame und korrigierende Erfahrungen in ihrer beschädigten Leiblichkeit erleben. Dafür gilt dem Autor ein ganz besonderer Dank.

Martin J. Waibel

DOI 10.2378/ktb2020.art22d

\title{
Renate Schwenk, Christine Pechtl (Hrsg.): Körper im Dialog. Theorie und Anwendungsfelder der Bioenergetischen Analyse
}

\author{
Psychosozial-Verlag, 2019, Gießen, 414 Seiten, 44,90€ (D)
}

U日: $\mathrm{m}$ es gleich vorweg zu schicken: Dieses Buch ist ein außerordentlich bereichernder Beitrag. Einerseits bietet es einen differenzierten, tiefen, vielschichtigen und plastischen Einblick in die Denkmodelle sowie Praxis von Bioenergetischer Analyse (BA) in nicht-therapeutischen Bereichen. Andererseits werden BA-Konzepte mit anderen, relevanten theoretischen und praxeologischen Ansätzen integriert betrachtet, die ihren Ursprung nicht im körperpsychotherapeutischen Feld haben. U.a. handelt es sich dabei um Konzepte aus der Feldforschung, theoretische Ansätze aus dem Konstruktivismus der Systemtheorie und Organisationsentwicklung, aber auch Ansätze aus Leadership-Science und Resilienzforschung. Das Buch ist aber keine praxeologische oder methodische/konzeptionelle Einführung in die BA.

Die Herausgeberin, die Österreichische Gesellschaft für körperbezogene Psychotherapie (DÖK), besteht seit 40 Jahren. Es wird aufgezeigt, wie die BA in unterschiedlichen Arbeits- feldern praktiziert und integriert wird und wie diese Erfahrungen und Erkenntnisse wieder in den Verein zurückgebracht werden. Ein solches Vorgehen sucht offensichtlich seinesgleichen im körperpsychotherapeutischen Feld. Allein schon der Ansatz, auch im nicht-therapeutischen Bereich substantiell und differenziert, aber auch kontextbezogen zu arbeiten, ist bereits eine Entdeckungsreise wert. Hinzu kommt das Unterfangen - äußerst erfolgreich dargestellt - BA-Theorie sowie -Konzepte, Ausbildung in BA, Entwicklung und Durchführung von Praxisprojekten sowie einen diesbezüglichen fruchtbaren, bereichernden Diskurs im Verein darzustellen. Hiervon lebt die Zukunft von BA und KPT, und die Arbeit ist dann nicht nur einem kleinen Kreis von ExpertInnen vorbehalten.

Im ersten Teil werden Aspekte der gemeinsamen Basis, wenn auch in kürzerer Form, so doch äußerst gehaltvoll, dargestellt. Es geht dabei um den historischen Abriss und die 